S. W. He, W. Q. Yang, R. Q. Yao and J. J. Wang

Nagoya Math. J.

Vol. 138 (1995), $51-64$

\title{
LOCAL TIMES OF SELF-INTERSECTION FOR MULTIDIMENSIONAL BROWNIAN MOTION*
}

\author{
SHENG-WU HE, WEN-QIANG YANG, RONG-QIN YAO \\ AND JIA-GANG WANG
}

We will define local times of self-intersection for multidimensional Brownian motion as generalized Wiener functionals under the framework of white noise analysis as in $\mathrm{H}$. Watanabe ([6]). By making use of the chaotic representation of $\delta$-function and precise computation we get a deep insight into the problem. In the section 1 multiple Wiener integrals with respect to multidimensional Brownian motion and chaotic representations for square-integrable Wiener functionals are given. They are indispensable, but seem not to be formulated clearly and correctly before. The useful concepts and results of white noise analysis are illustrated in the section 2 . Section 3 is the main part of the paper. The applications to local times are introduced in the section 4 briefly.

\section{Multiple Wiener integrals}

Let $\boldsymbol{B}=\left\{\left(B_{t}^{1}, \cdots, B_{t}^{d}\right),-\infty<t<\infty\right\}$ be a $d$-dimensional Brownian motion defined on a probability space $(\Omega, \mathscr{F}, \boldsymbol{P})$ such that $\mathscr{F}=\sigma\left\{\left(B_{t}^{1}, \cdots, B_{t}^{d}\right)\right.$, $-\infty<t<\infty\}$. We will define the multiple Wiener integrals with respect bo $\boldsymbol{B}$. The procedure will be sketched, and all proofs omitted, since they are completely similar to that for one-dimensional Brownian motion (cf. [3]).

Let $n=n_{1}+\cdots+n_{d}, n_{j} \geq 0, j=1, \ldots, d$. For each $f \in L^{2}\left(\boldsymbol{R}^{n}\right)$ define

$$
\hat{f}\left(t_{1}, \cdots, t_{n}\right)=\frac{1}{n_{1} ! \cdots n_{d} !} \sum_{\pi^{1}, \cdots, \pi^{d}} f\left(t_{\pi_{1}^{1}}, \cdots, t_{\pi_{n_{1}}^{1}}, \cdots, t_{\pi_{1}^{d}}, \cdots, t_{\pi_{n_{d}^{d}}}\right),
$$

where $\pi^{\mathrm{j}}, j=1, \ldots, d$, are the permutations of $\left(n_{1}+\cdots+n_{j-1}+1, \ldots, n_{1}+\right.$ $\left.\cdots+n_{j}\right), j=1, \ldots, d$, respectively, and the summation is over all permutations $\left\{\pi^{1}, \ldots, \pi^{d}\right\}$. Write

Received October 29, 1993.

*) The project supported by National Natural Science Foundation of China 


$$
\tilde{L}^{2}\left(\boldsymbol{R}^{n_{1}}\right) \otimes \cdots \otimes \hat{L}^{2}\left(\boldsymbol{R}^{n_{d}}\right)=\left\{\hat{f}: f \in L^{2}\left(\boldsymbol{R}^{n}\right)\right\} .
$$

For each $f \in L^{2}\left(\boldsymbol{R}^{n}\right)$ we can define

$$
I_{n_{1}, \ldots, n_{d}}(f)=\int f\left(t_{1}, \cdots, t_{n}\right) d B_{t_{n}}^{1} \cdots d B_{t_{n_{1}}}^{1} \cdots d B_{t_{n_{1}+\cdots+t_{n_{d-1}}+1}^{d}}^{d} \cdots d B_{t_{n}}^{d},
$$

such that for any $f \in L^{2}\left(\mathbf{R}^{n}\right)$

1) $I_{n_{1}, \cdots, n_{d}}(f)=I_{n_{1}, \cdots, n_{d}}(\hat{f})$,

2) $E\left[I_{n_{1}, \cdots, n_{d}}(f)\right]=0$,

3) for any $g \in L^{2}\left(\boldsymbol{R}^{m}\right), m=m_{1}+\cdots+m_{d}, m_{j} \geq 0, j=1, \ldots, d$,

$$
E\left[I_{n_{1}, \cdots, n_{d}}(f) I_{m_{1}, \cdots, m_{d}}(g)\right]=n_{1} ! \cdots n_{d} !\langle\bar{f}, \hat{g}\rangle \delta_{\left(n_{1}, \cdots, n_{d}\right)\left(m_{1}, \cdots, m_{d}\right)}
$$

4) for any $g \in L^{2}\left(\boldsymbol{R}^{n}\right), a, b \in \boldsymbol{R}$

$$
I_{n_{1}, \cdots, n_{d}}(a f+b g)=a I_{n_{1}, \cdots, n_{d}}(f)+b I_{n_{1}, \cdots, n_{d}}(g),
$$

5) for $f\left(t_{1}, \cdots, t_{n}\right)=f_{1}\left(t_{1}, \cdots, t_{n_{1}}\right) \cdots f_{d}\left(t_{n_{1}+\cdots+n_{d-1}+1}, \cdots, t_{n}\right), f_{j} \in L^{2}\left(\boldsymbol{R}^{n_{j}}\right)$,

$$
\begin{gathered}
j=1, \ldots, d, \\
I_{n_{1}, \cdots, n_{d}}(f)=\int f_{1} d B_{t_{1}}^{1} \cdots d B_{t_{n_{1}}}^{1} \cdots \int f_{d} d B_{t_{1}}^{d} \cdots d B_{t_{n_{d}}}^{d} .
\end{gathered}
$$

Thus $I_{n_{1}, \cdots, n_{d}}(\hat{f})$ is an isometric mapping from $\hat{L}^{2}\left(\boldsymbol{R}^{n_{1}}\right) \otimes \cdots \otimes \hat{L}^{2}\left(\boldsymbol{R}^{n_{d}}\right)$ into $\left(L^{2}\right)=L^{2}(\Omega, \mathscr{F}, \boldsymbol{P})$. The most important result is the following chaotic representation for square-integrable Wiener functionals:

To any $\phi \in\left(L^{2}\right)$ corresponds a unique sequence $\left\{\phi_{n_{1}, \cdots, n_{d}} \in \bar{L}^{2}\left(\boldsymbol{R}^{n_{1}}\right) \otimes \cdots\right.$ $\left.\otimes \hat{L}^{2}\left(\boldsymbol{R}^{n_{d}}\right), n, \geq 0, j=1, \ldots, d\right\}$ such that

$$
\phi=\sum_{n=0}^{\infty} \sum_{n_{1}+\cdots+n_{d}=n} I_{n_{1}, \cdots, n_{d}}\left(\phi_{n_{1}, \cdots, n_{d}}\right)
$$

and

$$
\|\phi\|_{2}^{2}=\sum_{n=0}^{\infty} \sum_{n_{1}+\cdots+n_{d}=n} n_{1} ! \cdots n_{d} !\left|\phi_{n_{1}, \cdots, n_{d}}\right|_{2}^{2}
$$

where $I_{0}(f)=E[f]$. Later we will denote (1.8) simply by $\phi \sim\left(\phi_{n_{1}, \cdots, n_{d}}\right)$.

\section{White noise space}

We adopt the framework of white noise analysis set by T. Hida (cf. [1] or [2]). 
Let $\mathscr{S}(R)$ be the Schwartz space of rapidly decreasing functions on $\boldsymbol{R}$. Denote by $A$ the self-adjoint extension of the harmonic oscillator operator on $L^{2}(\boldsymbol{R})$ :

$$
A f(t)=-f^{\prime \prime}(t)+\left(1+t^{2}\right) f(t), \quad f \in \mathscr{S}(\boldsymbol{R}) .
$$

Let $H_{n}(x), n \geq 0$, be the Hermite polynomial of order $n$, and

$$
e_{n}(x)=\left(n ! 2^{n}\right)^{-1 / 2}(\pi)^{-1 / 4} H_{n}(x) e^{-x^{2 / 2}}, n \geq 0 .
$$

Then $e_{n} \in \mathscr{S}(\boldsymbol{R})$, and $\left\{e_{n}, n \geq 0\right\}$ is an orthogonal normal basis of $L^{2}(\boldsymbol{R})$ and

$$
A e_{n}=(2 n+2) e_{n}, \quad n \geq 0 \text {. }
$$

Put

$$
\begin{gathered}
|f|_{2, p}^{2}=\left|A^{p} f\right|_{2}^{2}=\sum_{n=0}^{\infty}(2 n+2)^{2 p}\left|\left\langle f, e_{n}\right\rangle\right|^{2}, f \in L^{2}(\boldsymbol{R}), \\
\mathscr{\&}_{p}(\boldsymbol{R})=\mathscr{D}\left(A^{p}\right)=\left\{f \in L^{2}(\boldsymbol{R}):|f|_{2, p}^{2}<\infty\right\}, p \geq 0 .
\end{gathered}
$$

With $\left\{|\cdot|_{2, p}, p \geq 0\right\} \mathscr{S}(\boldsymbol{R})$ is a nuclear space. Let $\mathscr{S}^{\prime}(\boldsymbol{R})$ be its dual. Set

$$
\mathscr{\&}_{p}(\boldsymbol{R})=\left\{f \in \mathscr{S}^{\prime}(\boldsymbol{R}):|f|_{2, p}^{2}=\sum_{n=0}^{\infty}(2 n+2)^{2 p}\left|\left\langle f, e_{n}\right\rangle\right|^{2}<\infty\right\}, p \in \boldsymbol{R},
$$

where $\langle\cdot, \cdot\rangle$ denotes the pairing between $\mathscr{S}(\boldsymbol{R})$ and $\mathscr{S}^{\prime}(\boldsymbol{R})$. Then

$$
\mathscr{H}(\boldsymbol{R})=\bigcap_{p \in \boldsymbol{R}} \mathscr{\bigotimes}_{p}(\boldsymbol{R}), \quad \mathscr{S}^{\prime}(\boldsymbol{R})=\bigcup_{p \in \boldsymbol{R}} \mathscr{\bigotimes}_{p}(\boldsymbol{R}) .
$$

By Minlos theorem there exists a unique probability measure $\mu$ on $\mathscr{B}\left(\mathscr{S}^{\prime}(\boldsymbol{R})\right)$, the $\sigma$-field generated by cylinder sets, such that

$$
\int_{\mathscr{S}^{\prime}(\boldsymbol{R})} e^{i\langle x, \xi\rangle} \mu(d x)=\exp \left\{-\frac{1}{2}|\xi|_{2}^{2}\right\}, \quad \xi \in \mathscr{S}(\boldsymbol{R}) .
$$

The measure $\mu$ is called the white noise measure, and the probability space $\left(\mathscr{S}^{\prime}(\boldsymbol{R}), \mathscr{B}\left(\mathscr{S}^{\prime}(\boldsymbol{R})\right), \mu\right)$ is called the white noise space. Define

$$
\left(\Omega_{d}, \mathscr{F}_{d}, \mu_{d}\right)=\left(\mathscr{S}^{\prime}(\boldsymbol{R}), \mathscr{B}\left(\mathscr{S}^{\prime}(\boldsymbol{R})\right), \mu\right)^{d} .
$$

It will be our fundamental probability space, and denote by $\left(L^{2, d}\right)$ the $L^{2}$-space on it. Set

$$
X_{\xi}^{j}(x)=\left\langle x_{j}, \xi\right\rangle, \quad x=\left(x_{1}, \ldots, x_{d}\right) \in \Omega_{d}, \xi \in \&(\boldsymbol{R}), j=1, \ldots, d .
$$

For each $j, \xi \rightarrow X_{\xi}^{j}$ is an isometric mapping from $\&(\boldsymbol{R})$ into $\left(L^{2, d}\right)$, and can be extended as an isometric mapping from $L^{2}(\boldsymbol{R})$ into $\left(L^{2, d}\right)$, i.e., $X_{\xi}^{j}$ is well defined for 
$\xi \in L^{2}(\boldsymbol{R})$. Put

$$
f_{t}= \begin{cases}1_{(0, t]}, & t \geq 0, \\ 1_{(t, 0]}, & t<0,\end{cases}
$$

and

$$
B_{t}^{j}(x)=\left\langle x_{\jmath}, f_{t}\right\rangle, \quad-\infty<t<\infty, j=1, \ldots, d .
$$

Then $\boldsymbol{B}=\left\{\left(B_{t}^{1}, \ldots, B_{t}^{d}\right),-\infty<t<\infty\right\}$ is a $d$-dimensional Brownian motion, and $\mathscr{F}_{d}=\sigma\left\{\left(B_{t}^{1}, \cdots, B_{t}^{d}\right),-\infty<t<\infty\right\}$. Let $\phi \sim\left(\phi_{n_{1}, \cdots, n_{d}}\right) \in\left(L^{2, d}\right)$. For $p \geq$ 0 , define

$$
\begin{aligned}
& \left(\mathscr{S}^{d}\right)_{p}=\left\{\phi \sim\left(\phi_{n_{1}, \cdots, n_{d}}\right) \in\left(L^{2, d}\right):\|\phi\|_{2, p}^{2}=\right. \\
& \left.\sum_{n=0}^{\infty} \sum_{n_{1}+\cdots+n_{d}=n} n_{1} ! \cdots n_{d} !\left|\left(A^{p}\right)^{\otimes n} \phi_{n_{1}, \cdots, n_{d}}\right|_{2}^{2}<\infty\right\} .
\end{aligned}
$$

Denote by $\left(\&^{d}\right)_{-p}$ the dual of $\left(\varnothing^{d}\right)_{p}$. Define

$$
\left(\varnothing^{d}\right)=\bigcap_{p \geq 0}\left(\varnothing^{d}\right)_{p} .
$$

With $\left\{\|\cdot\|_{2, p}, p \geq 0\right\}\left(\mathscr{\bigotimes}^{d}\right)$ is a nuclear space, and its dual

$$
\left(\oiint^{d}\right)^{*}=\bigcup_{p \geq 0}\left(\oiint^{d}\right)_{-p} \text {. }
$$

Each element of $\left(\varnothing^{d}\right)$ is called a test functional, and each element of $\left(\varnothing^{d}\right)^{*}$ is called a generalized Wiener functional or Hida distribution.

For $\xi=\left(\xi_{1}, \ldots, \xi_{d}\right) \in \mathscr{S}^{d}(\boldsymbol{R})$, exponential functional

$$
\begin{gathered}
\mathscr{E}(\xi)(x)=\exp \left\{\sum_{j=1}^{d}\left(\left\langle x_{j}, \xi_{j}\right\rangle-\frac{1}{2}\left|\xi_{j}\right|_{2}^{2}\right)\right\} \sim\left(\frac{1}{n_{1} ! \cdots n_{d} !} \xi_{1}^{\otimes n_{1}} \otimes \cdots \otimes \xi_{d}^{\otimes n_{d}}\right), \\
x=\left(x_{1}, \cdots, x_{d}\right) \in \Omega_{d} .
\end{gathered}
$$

is a test functional. For any $\Phi \in\left(\mathscr{S}^{d}\right)^{*}$ the $S$-transform of $\Phi$ is defined as

$$
(S \Phi)(\xi)=\left\langle\langle\Phi, \mathscr{E}(\xi)\rangle, \quad \xi \in \mathscr{S}^{d}(\mathbf{R}),\right.
$$

where $\langle\langle\cdot, \cdot\rangle\rangle$ denotes the pairing between $\left(\&^{d}\right)$ and $\left(\&^{d}\right)^{*}$. If $\Phi \in\left(L^{2, d}\right)$, then

$$
(S \Phi)(\xi)=\int_{\Omega_{d}} \Phi(x+\xi) \mu_{d}(d x) .
$$

A functional $U$ on $\mathscr{S}^{d}(\boldsymbol{R})$ is called a $U$-functional. If

1) for each $\xi=\left(\xi_{1}, \ldots, \xi_{d}\right) \in \mathscr{S}^{d}(\boldsymbol{R})$ the mapping 


$$
\left(\lambda_{1}, \cdots, \lambda_{d}\right) \rightarrow U\left(\lambda_{1} \xi_{1}, \cdots, \lambda_{d} \xi_{d}\right)
$$

has analytic continuation, denoted by $u\left(z_{1}, \cdots, z_{d} ; \xi_{1}, \cdots, \xi_{d}\right)$, on $\boldsymbol{C}^{n}$;

2) for any $n=n_{1}+\cdots+n_{d}, n_{j} \geq 0, j=1, \ldots, d$,

$$
\begin{gathered}
U_{n_{1}, \cdots, n_{d}}\left(\xi_{1}, \cdots, \xi_{d}\right)=\frac{1}{n_{1} ! \cdots n_{d} !} \sum_{j_{1}=1}^{n_{1}} \cdots \sum_{j_{d}=1}^{n_{d}}(-1)^{n-\left(j_{1}+\cdots+j_{d}\right)} \\
\sum_{l_{1}^{1}<\cdots<l_{j_{1}}^{1}} \cdots \sum_{l_{1}^{d}<\cdots<l_{j_{d}}^{d}} \frac{\partial^{n}}{\partial^{n_{1}} z_{1} \cdots \partial^{n_{d}} z_{d}} u\left(0, \cdots, 0 ; \xi_{l_{1}^{1}}+\cdots+\xi_{l_{j_{1}^{1}}} \cdots, \xi_{l_{1}^{d}}+\cdots+\xi_{l_{j_{d}}}\right)
\end{gathered}
$$

is multilinear in $\left(\xi_{1}, \cdots, \xi_{d}\right)$;

3) there exist constants $C_{1}>0, C_{2}>0, p \in \boldsymbol{R}$ such that for all $\left(z_{1}, \cdots\right.$, $\left.z_{d}\right) \in C^{n}$ and $\left(\xi_{1}, \cdots, \xi_{d}\right) \in \mathscr{\wp}^{d}(\boldsymbol{R})$

$$
\left|u\left(z_{1}, \cdots, z_{d} ; \xi_{1}, \cdots, \xi_{d}\right)\right| \leq C_{1} \exp \left\{C_{2} \sum_{j=1}^{d}\left|z_{j}\right|^{2}\left|\xi_{j}\right|_{2, p}^{2}\right\} .
$$

Potthoff-Streit characterization theorem states that a functional on $\mathscr{\bigotimes}^{d}(\boldsymbol{R})$ is the $S$-transform of a Hida distribution if and only if it is a $U$-functional (cf. [5], only the case of $d=1$ is discussed there). Every Hida distribution is uniquely determined by its $S$-transform. In particular, if

$$
U(\xi)=\sum_{n=0}^{\infty} \sum_{n_{1}+\cdots+n_{d}=n}\left\langle f_{n_{1}, \cdots, n_{d}}, \xi_{1}^{\otimes n_{1}} \otimes \cdots \otimes \xi_{d}^{\otimes n_{d}}\right\rangle
$$

and for some $p \geq 0$

$$
\sum_{n=0}^{\infty} \sum_{n_{1}+\cdots+n_{d}=n} n_{1} ! \cdots n_{d} !\left|\left(A^{-p}\right)^{\otimes_{n}} f_{n_{1}, \cdots, n_{d}}\right|_{2}^{2}<\infty,
$$

where $f_{n_{1}, \cdots, n_{d}} \in \hat{L}^{2}\left(\boldsymbol{R}^{n_{1}}\right) \otimes \cdots \otimes \hat{L}^{2}\left(\boldsymbol{R}^{n_{d}}\right)$, then there exists $\Phi \in\left(\oiint^{d}\right)_{-p}$ such that $(S \Phi)(\xi)=U(\xi)$, and the expression in (2.17) is just equal to $\|\Phi\|_{2,-p}^{2}$. In this case, we also denote $\Phi \sim\left(f_{n_{1}, \cdots, n_{d}}\right)$.

\section{Local times of self-intersection}

Let $f$ be a bounded Borel function on $\boldsymbol{R}^{d}$. Then it is well-known by (2.15) that for any $0 \leq s \leq t$

$$
\left[S f\left(\boldsymbol{B}_{t}-\boldsymbol{B}_{s}\right)\right](\xi)=\left[T_{t-s}^{(d)} f\right]\left(\int_{s}^{t} \xi(r) d r\right),
$$

where $\left\{T_{t}^{(d)}, t \geq 0\right\}$ is the transition semigroup of $d$-dimensional Brownian mo- 
tion:

$$
\begin{aligned}
& {\left[T_{t-s}^{(d)} f\right](x)=} \\
& \quad \int f\left(y_{1}, \cdots, y_{d}\right) \frac{1}{[2 \pi(t-s)]^{d / 2}} \exp \left\{-\frac{1}{2(t-s)} \sum_{j=1}^{d}\left(y_{j}-x_{j}\right)^{2}\right\} d y_{1} \cdots d y_{d}
\end{aligned}
$$

Let $\delta_{\boldsymbol{a}}, \boldsymbol{a}=\left(a_{1}, \cdots, a_{d}\right)$, be the $\delta$-function at point $\boldsymbol{a}$. From (3.1) we have formally

$$
\begin{aligned}
& {\left[S \delta_{\boldsymbol{a}}\left(\boldsymbol{B}_{t}-\boldsymbol{B}_{s}\right)\right](\xi)=} \\
& \quad \frac{1}{[2 \pi(t-s)]^{d / 2}} \exp \left\{-\frac{1}{2(t-s)} \sum_{j=1}^{d}\left(a_{j}-\int_{s}^{t} \xi_{j}(r) d r\right)^{2}\right\} .
\end{aligned}
$$

It is not difficult to see that the right hand of (3.2) is a $U$-functional. In fact, by the formula of the generating function for Hermite polynomials we have

$$
\begin{aligned}
& \exp \left\{-\frac{1}{2(t-s)}\left(\int_{s}^{t} \xi_{j}(r) d r-a_{j}\right)^{2}\right\}=e^{-x_{j}^{2}} \sum_{n=0}^{\infty} \frac{1}{n !} H_{n}\left(x_{j}\right)\left(\frac{\int_{s}^{t} \xi_{j}(r) d r}{\sqrt{2(t-s)}}\right)^{n} \\
& {[2 \pi(t-s)]^{-d / 2} \exp \left\{-\frac{1}{2(t-s)} \sum_{j=1}^{d}\left(\int_{s}^{t} \xi_{j}(r) d r-a_{j}\right)^{2}\right\}} \\
& \quad=[2 \pi(t-s)]^{-d / 2} e^{-\sum_{j=1}^{d} x_{j}^{2}} \sum_{n=0}^{\infty} \sum_{n_{1}+\cdots+n_{d}=n} \prod_{j=1}^{d}\left\{\frac{1}{n_{j} !} H_{n_{j}}\left(x_{j}\right)\left(\frac{\int_{s}^{t} \xi_{j}(r) d r}{\sqrt{2(t-s)}}\right)^{n_{j}}\right\},
\end{aligned}
$$

where $x_{j}=\frac{a_{j}}{\sqrt{2(t-s)}}$. Put

$$
\begin{aligned}
\psi_{n_{1}, \cdots, n_{d}}^{(a)}(s, t) & =[2 \pi(t-s)]^{-d / 2} e^{-\sum_{j=1}^{d} x_{j}^{2}} \prod_{j=1}^{d} \frac{1}{n_{j} !} H_{n_{j}}\left(x_{j}\right)\left(\frac{1_{(s, t)}}{\sqrt{2(t-s)}}\right)^{\otimes n_{j}} \\
& =[2(t-s)]^{-d / 2} \pi^{-d / 4} e^{-\sum_{j=1}^{d} x_{j}^{2} / 2} \prod_{j=1}^{d} \frac{1}{\sqrt{n_{j} !}} e_{n_{j}}\left(x_{j}\right)\left(\frac{1_{(s, t]}}{\sqrt{t-s}}\right)^{\otimes n_{j}} .
\end{aligned}
$$

Note that $\left\{e_{n}(x), n \geq 0\right\}$ is uniformly bounded: $C=\sup _{n, x}\left|e_{n}(x)\right|<\infty$. Now for any $p>0$ we have

$$
\begin{aligned}
\mid\left(A^{-p}\right)^{\otimes n} \phi_{n_{1}, \cdots, n_{d}}^{(\boldsymbol{a})}(s, t) & \left.\right|_{2} ^{2} \leq 2^{-2 n p}\left|\phi_{n_{1}, \cdots, n_{d}}^{(\boldsymbol{a})}(s, t)\right|_{2}^{2} \\
& \leq 2^{-2 n p}[2(t-s)]^{-d} \pi^{-d / 2} \prod_{\jmath=1}^{d}\left(\frac{1}{n_{\jmath} !} e_{n_{j}}^{2}\left(x_{\jmath}\right)\right)\left|\frac{1_{(s, t]}}{\sqrt{t-s}}\right|_{2}^{2 n} \\
& \leq C^{2} 2^{-2 n p}[2(t-s)]^{-d} \pi^{-d / 2} \prod_{j=1}^{d} \frac{1}{n_{\jmath} !}
\end{aligned}
$$


where $n=n_{1}+\cdots+n_{d}$, and

$$
\begin{gathered}
\sum_{n=0}^{\infty} \sum_{n_{1}+\cdots+n_{d}=n} n_{1} ! \cdots n_{d} !\left|\left(A^{-p}\right)^{\otimes n} \phi_{n_{1}, \cdots, n_{d}}^{(\boldsymbol{a})}(s, t)\right|_{2}^{2} \\
\leq C^{2}[2(t-s)]^{-d} \pi^{-d / 2} \sum_{n=0}^{\infty} \sum_{n_{1}+\cdots+n_{d}=n} 2^{-2 n p} \\
\quad=C^{2}[2(t-s)]^{-d} \pi^{-d / 2} \sum_{n=0}^{\infty} 2^{-2 n p} C_{n+d-1}^{d-1}<\infty .
\end{gathered}
$$

According to (2.16) and (2.17), we obtain the following

Lemma 1. For any $0 \leq s<t, p>0$ and $\boldsymbol{a} \in \boldsymbol{R}^{d}$

$$
\delta_{\boldsymbol{a}}\left(\boldsymbol{B}_{t}-\boldsymbol{B}_{s}\right) \sim\left(\phi_{n_{1}, \cdots, n_{d}}^{(\boldsymbol{a})}(s, t)\right) \in\left(\oiint^{d}\right)_{-p}
$$

is a Hida distribution.

THEOREm 1. If $\boldsymbol{a} \neq \mathbf{0}$, then for any $t \geq 0, p>0$

$$
G_{d}^{\boldsymbol{a}}(t)=\int_{0 \leq u<v \leq t} \delta_{\boldsymbol{a}}\left(\boldsymbol{B}_{v}-\boldsymbol{B}_{u}\right) d u d v \in\left(\mathscr{\&}^{d}\right)_{-p}
$$

is a Hida distribution.

Proof. Noting that

$$
C_{\boldsymbol{a}}=\pi^{-d / 4} \sup _{u>0}\left\{(2 u)^{-d / 2} e^{-|\boldsymbol{a}|^{2} / 4 u}\right\}<\infty,
$$

(3.4) can be modified as

$$
\left|\left(A^{-p}\right)^{\otimes n} \phi_{n_{1}, \cdots, n_{d}}^{(\boldsymbol{a})}(s, t)\right|_{2}^{2} \leq C^{2} C_{\boldsymbol{a}^{2}}^{2} 2^{-2 n p} \prod_{j=1}^{d} \frac{1}{n_{j} !} .
$$

Then we have

$$
\begin{gathered}
\left\|\delta_{\boldsymbol{a}}\left(\boldsymbol{B}_{t}-\boldsymbol{B}_{s}\right)\right\|_{2,-p}^{2} \leq C^{2} C_{\boldsymbol{a}}^{2} \sum_{n=0}^{\infty} 2^{-2 n p} C_{n+d-1}^{d-1}, \\
\int_{0 \leq u<v \leq t}\left\|\delta_{\boldsymbol{a}}\left(\boldsymbol{B}_{v}-\boldsymbol{B}_{u}\right)\right\|_{2,-p} d u d v<\infty
\end{gathered}
$$

Hence $G_{d}^{(\boldsymbol{a})}(t) \in\left(\mathscr{\wp}^{d}\right)_{-p}$.

Theorem 2. For any $t \geq 0$ 


$$
G_{d}(t)=\int_{0 \leq u<v \leq t}\left[\delta\left(\boldsymbol{B}_{v}-\boldsymbol{B}_{u}\right)-\sum_{n=0}^{d-2} \sum_{n_{1}+\cdots+n_{d}=n} I_{n_{1}, \cdots, n_{d}}\left(\phi_{n_{1}, \cdots, n_{d}}(u, v)\right)\right] d u d v
$$

is a Hida distribution, called local times of self-intersection for $d$-dimensional Brownian motion, where $\delta=\delta_{\mathbf{0}}$.

Proof. At first, observe that let $p>0$

$$
\begin{aligned}
\left|A^{-p} 1_{(s, t]}\right|_{2}^{2} & =\sum_{k=0}^{\infty}(2 k+2)^{-2 p}\left|\left\langle 1_{(s, t]}, e_{k}\right\rangle\right|^{2} \\
& \leq \sum_{k=0}^{\infty}(2 k+2)^{-2 p}(t-s)^{2} \sup _{x} e_{k}^{2}(x)
\end{aligned}
$$

Since $\sup _{x} e_{k}^{2}(x)=O\left(k^{-\frac{1}{6}}\right)$, take $p$ large enough such that

$$
\sum_{k=0}^{\infty}(2 k+2)^{-2 p} \sup _{x} e_{k}^{2}(x)<1
$$

Then by (3.6) and (3.7) we get

$$
\begin{aligned}
\left|\left(A^{-p}\right)^{\otimes n} \psi_{n_{1}, \cdots, n_{d}}(s, t)\right|_{2}^{2} & =[2 \pi(t-s)]^{-d} \prod_{j=1}^{d}\left(\frac{H_{n_{j}}(0)}{n_{j} !}\right)^{2}\left|A^{-p} \frac{1_{(s, t]}}{\sqrt{2(t-s)}}\right|_{2}^{2 n} \\
& \leq \frac{1}{n_{1} ! \cdots n_{d} !}(2 \pi)^{-d}(t-s)^{n-d}
\end{aligned}
$$

noting that $H_{2 k+1}(0)=0, H_{2 k}(0)=(-1)^{k} \frac{(2 k) !}{k !}, k \geq 0$, for any $k \geq 0$, $H_{k}^{2}(0) \leq k ! 2^{k}$. Let $\alpha>0$, from (3.8) we have

$$
\begin{aligned}
\| \delta & \left(\boldsymbol{B}_{t}-\boldsymbol{B}_{s}\right)-\sum_{n=0}^{d-2} \sum_{n_{1}+\cdots+n_{d}=n} I_{n_{1}, \cdots, n_{d}}\left(\phi_{n_{1}, \cdots, n_{d}}(s, t)\right) \|_{2,-(p+\alpha)}^{2} \\
& =\left\|\sum_{n=d-1}^{\infty} \sum_{n_{1}+\cdots+n_{d}=n} I_{n_{1}, \cdots, n_{d}}\left(\psi_{n_{1}, \cdots, n_{d}}(s, t)\right)\right\|_{2,-(p+\alpha)}^{2} \\
& \left.=\sum_{n=d-1}^{\infty} \sum_{n_{1}+\cdots+n_{d}=n} n_{1} ! \cdots n_{d} ! \mid\left(A^{-(p+\alpha)}\right)^{\otimes_{n}} \phi_{n_{1}, \cdots, n_{d}}(s, t)\right)\left.\right|_{2} ^{2} \\
& \left.\leq \sum_{n=d-1}^{\infty} \sum_{n_{1}+\cdots+n_{d}=n} n_{1} ! \cdots n_{d} ! 2^{-2 n \alpha} \mid\left(A^{-p}\right)^{\otimes_{n}} \phi_{n_{1}, \cdots, n_{d}}(s, t)\right)\left.\right|_{2} ^{2} \\
& \leq \sum_{n=d-1}^{\infty} \sum_{n_{1}+\cdots+n_{d}=n} 2^{-2 n \alpha}(2 \pi)^{-d}(t-s)^{n-d} \\
& =(2 \pi)^{-d} \sum_{n-d-1}^{\infty} 2^{-2 n \alpha} C_{n+d-1}^{d-1}(t-s)^{n-d} .
\end{aligned}
$$


Take $\alpha$ large enough such that $2^{-\alpha} \sqrt{t}<1$. Then

$$
\int_{0 \leq u<v \leq t}\left\|\delta\left(\boldsymbol{B}_{v}-\boldsymbol{B}_{u}\right)-\sum_{n=0}^{d-2} \sum_{n_{1}+\cdots+n_{d}=n} I_{n_{1}, \cdots, n_{d}}\left(\phi_{n_{1}, \cdots, n_{d}}(u, v)\right)\right\|_{2,-(p+\alpha)} d u d v<\infty .
$$

Hence, the integral in the right hand of (3.5) exists in $\left(\mathscr{\bigotimes}^{d}\right)_{-(p+\alpha)}$ as Bochner integral, i.e., $G_{d}(t) \in\left(S^{d}\right)_{-(p+\alpha)}$ is a Hida distribution.

From (3.5) we have

$$
\begin{aligned}
G_{1}(t)= & \int_{0 \leq u<v \leq t} \delta\left(\boldsymbol{B}_{v}-\boldsymbol{B}_{u}\right) d u d v \\
G_{2}(t)= & \int_{0 \leq u<v \leq t}\left\{\delta\left(\boldsymbol{B}_{v}-\boldsymbol{B}_{u}\right)-[2 \pi(v-u)]^{-1}\right\} d u d v \\
G_{3}(t)= & \int_{0 \leq u<v \leq t}\left\{\delta\left(\boldsymbol{B}_{v}-\boldsymbol{B}_{u}\right)-[2 \pi(v-u)]^{-3 / 2}\right\} d u d v, \\
G_{4}(t)= & \int_{0 \leq u<v \leq t}\left\{\delta\left(\boldsymbol{B}_{v}-\boldsymbol{B}_{u}\right)-\frac{1}{[2 \pi(v-u)]^{2}}\right. \\
G_{5}(t)= & \int_{0 \leq u<v \leq t}\left\{\delta\left(\boldsymbol{B}_{v}-\boldsymbol{B}_{u}\right)-\frac{1}{(2 \pi)^{2}(v-u)^{3}} \sum_{j=1}^{4} \int_{u}^{v}\left(B_{r}^{j}-B_{u}^{j}\right) d B_{r}^{j}\right\} d u d v, \\
& \left.+\frac{1}{(2 \pi)^{5 / 2}(v-u)^{7 / 2}} \sum_{j=1}^{5} \int_{u}^{r}\left(B_{r}^{j}-B_{u}^{j}\right) d B_{r}^{j}\right\} d u d v
\end{aligned}
$$

For $d \geq 2$, the integral

$$
\int_{0 \leq u<v \leq t} \delta\left(\boldsymbol{B}_{v}-\boldsymbol{B}_{u}\right) d u d v
$$

has no meaning even in generalized sense, and must be renormalized according to (3.5). The renormalization parts in $G_{4}(t)$ and $G_{5}(t)$ in Watanabe ([6]) are mistaken, caused by his small error in computation.

Lemma 2. Set

$$
a_{n}^{(d)}(t)=\left|\int_{0 \leq u<v \leq t}(v-u)^{-\left(n+\frac{d}{2}\right)} 1_{(u, v]}^{\otimes 2 n} d u d v\right|_{2}^{2} .
$$


Then as $n \rightarrow \infty$, for $t>0$ we have

$$
a_{n}^{(d)}(t) \begin{cases}\sim \frac{2 t^{3}}{3 n^{2}}, & d=1, \\ \sim \frac{2 t^{2}}{n^{2}}, & d=2, \\ =\infty, & d \geq 3 .\end{cases}
$$

Proof. By Fubini theorem on interchanging the order of integration, we have

$$
\begin{aligned}
& a_{n}^{(d)}(t)=\int_{\substack{0 \leq u<v \leq t \\
0 \leq r<s \leq t}} \frac{\left|\left\langle 1_{(u, v)}, 1_{(r, s)}\right\rangle\right|^{2 n}}{(v-u)^{n+d / 2}(s-r)^{n+d / 2}} d u d v d r d s \\
& =\int_{\substack{0 \leq u<v \leq t, 0 \leq r<s \leq t, r<u<s \text { or } u<r<v}} \frac{(v \wedge s-u \vee r)^{2 n}}{(v-u)^{n+d / 2}(s-r)^{n+d / 2}} d u d v d r d s \\
& =2 \int_{0 \leq r<u<s<v \leq t} \frac{(s-u)^{2 n}}{(v-u)^{n+d / 2}(s-r)^{n+d / 2}} d u d v d r d s \\
& +2 \int_{0 \leq r<u<v<s \leq t} \frac{(v-u)^{n-d / 2}}{(s-r)^{n+d / 2}} d u d v d r d s \\
& =2\left(a_{n 1}^{(d)}(t)+a_{n 2}^{(d)}(t)\right) . \\
& a_{n 2}^{(d)}(t)=\int_{0 \leq r<s \leq t}(s-r)^{-(n+d / 2)} d r d s \int_{r<u<v<s}(v-u)^{n-d / 2} d u d v \\
& =[(n-d / 2+1)(n-d / 2+2)]^{-1} \int_{0 \leq r<s \leq t}(s-r)^{2-d} d r d s \\
& =C_{t}^{(d)}[(n-d / 2+1)(n-d / 2+2)],
\end{aligned}
$$

where

$$
C_{t}^{(d)}=\int_{0 \leq r<s \leq t}(s-r)^{-d+2} d r d s= \begin{cases}t^{3} / 6, & d=1 \\ t^{2} / 2, & d=2 \\ \infty, & d \geq 3\end{cases}
$$

Below we consider only the case of $d=1$ or 2 .

$$
a_{n 1}^{(d)}(t)=2 \int_{0 \leq u<s<v \leq t} \frac{(s-u)^{2 n}}{(v-u)^{n+d / 2}} d u d v d s \int_{0}^{u} \frac{d r}{(s-r)^{n+d / 2}}
$$




$$
\begin{gathered}
=(n+d / 2-1)^{-1} \int_{0 \leq u<s<v \leq t}\left[\frac{(s-u)^{n-d / 2+1}}{(v-u)^{n+d / 2}}-\frac{(s-u)^{2 n}}{(v-u)^{n+d / 2} s^{n+d / 2-1}}\right] d u d v d s \\
=(n+d / 2-1)^{-1}\left[a_{n 11}^{(d)}(t)-a_{n 12}^{(d)}(t)\right] .\left(0 \leq a_{n 12}^{(d)}(t) \leq a_{n 11}^{(d)}(t) .\right)
\end{gathered}
$$

$$
\begin{aligned}
& a_{n 11}^{(d)}(t)=\int_{0 \leq u<v \leq t}(v-u)^{-(n+d / 2)} d u d v \int_{u}^{v}(s-u)^{n-d / 2+1} d s \\
&=C_{t}^{(d)}(n-d / 2+2)^{-1} \cdot \\
& a_{n 12}^{(d)}(t)=\int_{0 \leq u<s \leq t} \frac{(s-u)^{2 n}}{s^{n+d / 2-1}} d u d s \int_{s}^{t} \frac{d v}{(v-u)^{n+d / 2}}
\end{aligned}
$$$$
=(n+d / 2-1)^{-1} \int_{0 \leq u<s \leq t}\left[\frac{(s-u)^{n-d / 2+1}}{s^{n+d / 2-1}}-\frac{(s-u)^{2 n}}{s^{n+d / 2-1}(t-u) s^{n+d / 2-1}}\right] d u d s
$$$$
=(n+d / 2-1)^{-1}\left[a_{n 121}^{(d)}(t)-a_{n 122}^{(d)}(t)\right] .\left(0 \leq a_{n 122}^{(d)}(t) \leq a_{n 121}^{(d)}(t) .\right)
$$

$$
\begin{aligned}
a_{n 121}^{(d)}(t) & =\int_{0}^{t} s^{-(n+d / 2-1)} d s \int_{0}^{s}(s-u)^{n+d / 2+1} d u \\
& =(n+d / 2+1)^{-1} \int_{0}^{t} s^{-d+3} d s=o(1) .
\end{aligned}
$$

Thus $a_{n 122}^{(d)}(t)=o(1), a_{n 12}^{(d)}(t)=o(1)$, and by (3.19), (3.20) we have

$$
a_{n 1}^{(d)}(t)=\frac{1}{n^{2}} C_{t}^{(d)}(1+o(1)), \quad d=1,2 .
$$

At last, (3.15) follows from (3.16), (3.17), (3.18) and (3.21).

In Lemma 2 one needs only that $2 n$ is an integer.

Theorem 3. For any $t \geq 0$ and $\boldsymbol{a} \in \boldsymbol{R}$

$$
G_{d}^{(a)}(t) \in\left(L^{2, d}\right), \quad d=1,2 .
$$

Proof. From (3.3) we have

$$
G_{d}^{(a)}(t) \sim\left(\int_{0 \leq u<v \leq t} \frac{\exp \left(-\sum_{j=1}^{d} x_{j}^{2}\right)}{(2 \pi)^{2 / d}} \prod_{j=1}^{d} \frac{e_{n_{j}}\left(x_{j}\right)}{\sqrt{n_{j} !}} \frac{1_{(u, v]}^{\otimes n}}{(v-u)^{(n+d) / 2}} d u d v\right),
$$

where $x_{j}=\frac{a_{j}}{\sqrt{2(v-u)}}$ and the first term is considered as zero when $\boldsymbol{a}=\mathbf{0}$ and $d=2$. Hence, 


$$
\begin{aligned}
& \left\|G_{d}^{(a)}(t)\right\|_{2}^{2} \\
& \quad=\sum_{n=n_{0}}^{\infty} \sum_{n_{1}+\cdots+n_{d}=n}\left|\int_{0 \leq u<v \leq t} \frac{e^{-\sum_{j=1}^{d} x_{j}^{2}}}{(2 \pi)^{d / 2}} \prod_{j=1}^{d} e_{n_{j}}^{2}\left(x_{j}\right) \frac{1_{(u, v]}^{\otimes_{n}}}{(v-u)^{(n+d) / 2}} d u d v\right|_{2}^{2} \\
& \leq C\left(1+\sum_{n=1}^{\infty} \sum_{n_{1}+\cdots+n_{d}=n}(2 \pi)^{-d} \prod_{j=1}^{d}\left(n_{j}\right)^{-1 / 6} a_{n / 2}^{(d)}(t)\right) \\
& \leq C\left(1+\sum_{n=1}^{\infty} \sum_{n_{1}+\cdots+n_{d}=n} n^{-2} \prod_{j=1}^{d} n_{j}^{-1 / 6}\right) \\
& \leq C\left(1+\sum_{n=1}^{\infty} \sum_{n_{1}+\cdots+n_{d}=n} \prod_{j=1}^{d} n_{j}^{-1 / 6} n_{j}^{-1}\right) \\
& \leq C\left(1+\sum_{n=1}^{\infty} n^{-7 / 6}\right)^{d}<\infty,
\end{aligned}
$$

where

$$
n_{0}=\left\{\begin{array}{l}
1, a=0 \text { and } d=2 \\
0, \text { otherwise }
\end{array}\right.
$$

and $C$ is a constant depending on only $d$ and $t$, but may vary in different expressions. Thus $G_{d}^{(a)}(t) \in\left(L^{2, d}\right)$ for $d=1,2$.

Based on Lemma 2, it is plausible to reason that Theorem 3 does not hold for $d \geq 3$.

THEOREM 4. For any bounded Borel function $f$ and $t>0$

$$
\int_{0 \leq u<v \leq t} f\left(\boldsymbol{B}_{v}-\boldsymbol{B}_{u}\right) d u d v= \begin{cases}\int_{\boldsymbol{R}} f(a) G_{1}^{(a)}(t) d a, & d=1, \\ \int_{\boldsymbol{R}^{2}} f(\boldsymbol{a}) G_{2}^{(\boldsymbol{a})}(t) d \boldsymbol{a}, & d=2 .\end{cases}
$$

(3.22) is the so-called Tanaka's formula.

Proof. We only give the proof for $d=2$. We show the $S$-transforms of the

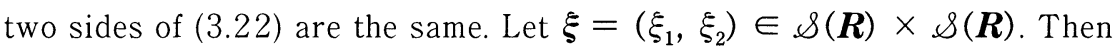

$$
\begin{aligned}
& {\left[S\left(\int_{\boldsymbol{R}^{2}} f(\boldsymbol{a}) G_{2}^{(\boldsymbol{a})}(t) d \boldsymbol{a}\right)\right](\xi)=\int_{\boldsymbol{R}^{2}} f(\boldsymbol{a})\left[S\left(G_{2}^{(\boldsymbol{a})}(t)\right)\right](\xi) d \boldsymbol{a} } \\
= & \int_{\boldsymbol{R}^{2}} \int_{0 \leq u<v \leq t} \frac{f(\boldsymbol{a})}{2 \pi(v-u)} \exp \left\{-\frac{1}{2(v-u)} \sum_{j=1}^{2}\left(a_{j}-\int_{u}^{v} \xi_{j}(r) d r\right)^{2}\right\} d u d v d \boldsymbol{a} \\
= & \int_{0 \leq u<v \leq t}\left[T_{v-u}^{(2)} f\right]\left(\int_{u}^{v} \xi(r) d r\right) d u d v .
\end{aligned}
$$


On the other hand,

$$
\begin{aligned}
{\left[S\left(\int_{0 \leq u<v \leq t} f\left(\boldsymbol{B}_{v}-\boldsymbol{B}_{u}\right) d u d v\right)\right](\xi)=} & \int_{0 \leq u<v \leq t}\left[S\left(f\left(\boldsymbol{B}_{v}-\boldsymbol{B}_{u}\right)\right](\xi) d u d v\right. \\
& =\int_{0 \leq u<v \leq t}\left[T_{v-u}^{(2)} f\right]\left(\int_{u}^{v} \xi(r) d r\right) d u d v
\end{aligned}
$$

Hence (3.22) follows.

Even for $d \geq 3$ the Tanaka's formula (3.22) holds, but the integrals in the right side of (3.22) should be understood in the sense of the Bochner integral in $\left(S^{\mathrm{d}}\right)_{-p}$ for any $p>0$.

\section{Local times}

Compared with the results and proofs in the above section, we can easily obtain the following results:

1) For any $d \geq 1, t \geq 0$

$$
L_{d}(t)=\int_{0}^{t}\left[\delta\left(\boldsymbol{B}_{u}\right)-\sum_{n=0}^{d-2} \sum_{n_{1}+\cdots+n_{d}=n} I_{n_{1}, \cdots, n_{d}}\left(\phi_{n_{1}, \cdots, n_{d}}(0, u)\right)\right] d u \in\left(\mathscr{S}^{d}\right)^{*} .
$$

Naturally, $L_{d}(t)$ may be considered as the local times at 0 .

2) For any $t>0, L_{1}(t) \in\left(L^{2,1}\right)$. In fact, note that

$$
\left|\int_{0}^{t} u^{-(n+1 / 2)} 1_{(0, u]}^{\otimes 2 n} d u\right|_{2}^{2}= \begin{cases}\frac{4 t}{2 n+1}, & d=1, \\ \infty, & d \geq 2 .\end{cases}
$$

So even for $d=2, L_{2}(t), t>0$, are impossible to be ordinary Wiener functionals.

3) If $\boldsymbol{a} \neq \mathbf{0}$, then for any $t \geq 0, p>0$

$$
L_{d}^{(\boldsymbol{a})}(t)=\int_{0}^{t} \delta_{\boldsymbol{a}}\left(\boldsymbol{B}_{u}\right) d u \in\left(\mathscr{\wp}^{d}\right)_{-p}
$$

is a Hida distribution. $L_{d}^{(\boldsymbol{a})}(t)$ may also be considered as the local time at $\boldsymbol{a}$.

4) For any $t>0, a \in \boldsymbol{R}, L_{1}^{(a)}(t) \in\left(L^{2,1}\right)$. In fact, noting that

$$
\int_{0 \leq u, v \leq t}\left|\left\langle\frac{1_{(0, u)}}{\sqrt{u}}, \frac{1_{(0, v)}}{\sqrt{v}}\right\rangle\right|^{n} d u d v=\frac{2 t^{2}}{n+2},
$$

it seems that for $\boldsymbol{a} \neq \mathbf{0}, L_{2}^{(\boldsymbol{a})}(t)$ may not be ordinary Wiener functionals.

For the case of $d=1$ we can give another treatment. Kubo has established 
the following generalized Ito's formula (cf. [4]): for all $f \in \mathscr{S}^{\prime}(\boldsymbol{R}), 0<s<t$,

$$
f\left(\boldsymbol{B}_{t}\right)-f\left(\boldsymbol{B}_{s}\right)=\int_{s}^{t} \partial_{u}^{*} f^{\prime}\left(\boldsymbol{B}_{u}\right) d u+\frac{1}{2} \int_{s}^{t} f^{\prime \prime}\left(\boldsymbol{B}_{u}\right) d u .
$$

Take $f(u)=u 1_{(a, \infty)}(u)=(u-a)^{+}$, then $f^{\prime}=1_{(a, \infty)}, f^{\prime \prime}=\delta_{a}$. Substituting them into (4.1) yields

$$
\left(\boldsymbol{B}_{t}-a\right)^{+}-\left(\boldsymbol{B}_{s}-a\right)^{+}=\int_{s}^{t} \partial_{u}^{*} 1_{(a, \infty)}\left(\boldsymbol{B}_{u}\right) d u+\frac{1}{2} \int_{s}^{t} \delta_{a}\left(\boldsymbol{B}_{u}\right) d u .
$$

Since $1_{(a, \infty)}\left(\boldsymbol{B}_{u}\right)$ is adapted, letting $s \rightarrow 0$ in (4.2) yields

$$
L_{1}^{(a)}(t)=\int_{s}^{t} \delta_{a}\left(\boldsymbol{B}_{u}\right) d u=2\left[\left(\boldsymbol{B}_{t}-a\right)^{+}-(-a)^{+}-\int_{0}^{t} 1_{(a, \infty)}\left(\boldsymbol{B}_{u}\right) d \boldsymbol{B}_{u}\right] \in\left(L^{2,1}\right) .
$$

This is just the ordinary definition of local times for one-dimensional Brownian motion. Obviously, we provide indeed a white noise analysis treatment of local times for one-dimensional Brownian motion. This approach applies also to local times of self-intersection for one-dimensional Brownian motion. In fact, by using (4.1) it is easy to get

$$
\begin{aligned}
& \int_{0 \leq u<v \leq t} \delta_{a}\left(\boldsymbol{B}_{v}-\boldsymbol{B}_{u}\right) d u d v \\
& =2\left[\int_{0}^{t}\left(\boldsymbol{B}_{t}-\boldsymbol{B}_{u}-a\right)^{+} d u-(-a)^{+} t-\int_{0 \leq u<v \leq t} 1_{(a, \infty)}\left(\boldsymbol{B}_{v}-\boldsymbol{B}_{u}\right) d u d \boldsymbol{B}_{v}\right] \in\left(L^{2,1}\right) .
\end{aligned}
$$

\section{REFERENCES}

[1] Hida, T., Analysis of Brownian Functionals, Carleton Math. Lecture, 13, 1975.

[2] Hida, T. and Potthoff, J., White noise analysis-an overview, In T. Hida et al. (eds.) White Noise Analysis-Mathematics and Applications, World Scientific, Singapore, 1990

[ 3 ] Ito, K., Multiple Wiener integrals, J. Math. Soc, Japan, 3 (1951), 157-169.

[4] Kubo, I, Ito formula for generalized Brownian functionals, Lecture Notes in Control and Information Science, No. 49 (1983), 156-166, Springer-Verlag.

[5] Potthoff, J. and Streit L., A characterization on Hida distributions, J. Funct. Anal., 101 (1991), 212-229.

[6] Watanabe, H., The local time of self-intersections of Brownian motion as generalized Brownian functionals, Lett. Math. Phys., 23 (1991), 1-9.

S. He, W. Yang and R. Yao

Department of Mathematical Statistics

East China Normal University

Shanghai, 200062 China
J. Wang

Institute of Applied Mathematics

East China University of Technology

Shanghai, 200237 China 